\title{
AC Transport Loss in Superconductors Carrying Harmonic Current with Different Phase Angles for Large Scale Power Components
}

\author{
Mohammad Yazdani-Asrami, Member, IEEE, Wenjuan Song, Member, IEEE, Min Zhang, Member, \\ IEEE, Weijia Yuan, Senior Member, IEEE, and Xiaoze Pei
}

\begin{abstract}
It is of great industrial interest and academic importance to investigate current harmonics impacts on AC losses of superconductors, especially in large scale power devices. However, only effect of amplitude of in-phase current harmonics on AC loss has been studied in literatures. We numerically characterized nonsinusoidal AC loss of superconducting tape carrying harmonic currents with orders below $20^{\text {th }}$ versus phase angles. A drastic AC loss variation was found when phase angle was considered for harmonic components. We observed that different harmonic orders show different $\mathrm{AC}$ loss profile versus phase angle.
\end{abstract}

Index Terms - AC loss, Harmonic phase angle, HTS tapes, Nonsinusoidal current.

\section{INTRODUCTION}

$\mathrm{S}$ UPERCONDUCTIVITY is a key technology for modernizing large scale power components, since high temperature superconducting (HTS) devices will bring many advantages over their conventional counterparts, such as higher efficiency, less energy consumption, compactness, lighter weight, and lower loss. HTS technology helps manufacture transformers, cables, motors, and generators with higher current density, and higher power to weight ratio and torque to weight ratio [1-4].

AC loss in HTS windings is one of the most critical aspects to consider during R\&D stage for any large scale HTS electric application. AC loss, as a source of heat load, is closely linked with the thermal load and efficiency of cryogenic cooling system, which adjusts and maintains the operating temperature of HTS components [5-6]. It is vital to accurately estimate AC losses in HTS devices under real operating conditions for designing efficient and reliable cooling system.

Nowadays, AC/DC converters and nonlinear loads are widely used in power grids, industries, and also in different transportation systems, including aerospace, railway, and naval applications [7-10]. Current harmonics, therefore, are inevitably produced inside power system, with different harmonic orders, amplitudes, and phase angles as compared to

M. Yazdani-Asrami, M. Zhang, and W. Yuan are with Department of Electronic and Electrical Engineering, University of Strathclyde, Glasgow, G1 1XW, U.K. (e-mail: m.yazdaniasrami@gmail.com, mohammad.yazdaniasrami@strath.ac.uk,min.zhang@strath.ac.uk,weijia.yuan@strath.ac.uk). the sinusoidal current waveform at fundamental frequency. Thus, it is important to precisely calculate AC losses in superconducting devices under nonsinusoidal condition. A number of previous literatures reported the nonsinusoidal AC loss analysis of HTS tapes under current harmonics at different harmonic orders, different amplitudes and total harmonic distortions (THDs) [11-17]. However, researchers merely considered the in-phase harmonic current with fundamental current, and effect of phase angle of harmonic current on $\mathrm{AC}$ loss behavior needs to be addressed.

In this paper, AC transport loss in a typical HTS tape carrying distorted current was modeled by $H$-formulation in COMSOL Multiphysics. The nonsinusoidal AC loss was calculated at different carrying current levels, current harmonic amplitudes, THDs, and different harmonic phase angles. All odd harmonic orders below $20^{\text {th }}$ were considered in this work.

\section{NUMERICAL CALCULATION METHOD}

All calculations were realized using a 2D model in COMSOL Multiphysics based on $H$-formulation [18-20]. Maxwell equations, Ohms law, and constitutive law were combined to solve the electromagnetic problem in an HTS tape carrying harmonic currents, as follows:

$$
\begin{gathered}
\nabla \times \boldsymbol{E}=-\partial \boldsymbol{B} / \partial t \\
\nabla \times \boldsymbol{H}=\boldsymbol{J} \\
\boldsymbol{E}=\rho \boldsymbol{J} \\
\boldsymbol{B}=\mu_{0} \mu_{r} \boldsymbol{H} \\
\boldsymbol{E}=E_{0}\left(\boldsymbol{J} / J_{\mathrm{c}}\right)^{n}
\end{gathered}
$$

where, $\boldsymbol{E}$ is electric field, $\boldsymbol{B}$ is magnetic flux density, $\boldsymbol{H}$ is magnetic field, $\boldsymbol{J}$ is current density, $\rho$ is resistivity, $\mu_{0}$ is permeability of free space, $\mu_{\mathrm{r}}$ is relative permeability, $\boldsymbol{E}_{0}=1$ $\mu \mathrm{V} / \mathrm{cm}, J_{\mathrm{c}}$ is critical current density, and $n$ is power law index.

The governing equation for solving this electromagnetic problem is expressed as follows [18-20]:
W. Song, and X. Pei are with the Department of Electronic \& Electrical Engineering, The University of Bath, Bath, BA2 7AY, U.K. (e-mails: ws603@bath.ac.uk, x.pei@bath.ac.uk). 


$$
\partial\left(\mu_{0} \mu_{r} \boldsymbol{H}\right) / \partial t+\nabla \times(\rho \nabla \times \boldsymbol{H})=0
$$

The AC loss $Q$ in the domain $\Omega$ is derived as [18-20]:

$$
\boldsymbol{Q}=2 \int_{T / 2}^{T} \int_{S} \boldsymbol{E} \cdot \boldsymbol{J} \mathrm{d} \Omega d t
$$

where $S$ and $T$ are the cross section of tape and period of one cycle of applied current, respectively. The AC loss is calculated by the integral of power density in the second half cycle, knowing that it is from the second half cycle that the magnetization process reaches a steady state.

Table I lists the specifications of the HTS tape used in this paper [21-22]. The nonsinusoidal current considered here is composed of fundamental current component, and a superimposed current harmonic with order $k=\{3,5,7,9,11$, $13,15,17,19\}$. THD is defined as the distortion level of transport current by each harmonic current component:

$$
T H D_{k}=I_{h k} / I_{h 1}
$$

where $T H D_{k}=\{0.1,0.2,0.3,0.4$, and 0.5$\} . I_{h k}$ is amplitude of each current harmonic, and $I_{\mathrm{h} 1}$ represents the amplitude of fundamental current harmonic; here, $I_{\mathrm{h} 1}$ was expressed as:

$$
I_{h 1}=i_{m} \cdot I_{\mathrm{c}}
$$

where $I_{\mathrm{c}}$ is the critical current of tape and $i_{\mathrm{m}}=\{0.1,0.2,0.3,0.4$, and 0.5 , indicating $10 \%$ to $50 \%$ of tape $I_{c}$.

To study the effect of phase angle of current harmonics on $\mathrm{AC}$ loss, the initial phase angle of fundamental current was kept as $0^{\circ}$ in the calculation, whilst phase angle of each current harmonic, $\varphi_{\mathrm{k}}$ was varied from $0^{\circ}$ to $360^{\circ}$ (i.e., 0 to $2 \pi$ ) and simulated by every $10^{\circ}$. The instantaneous applied nonsinusoidal current, $i_{\text {nonsin }}(\mathrm{t})$ is formulated as follows:

$$
i_{\text {nonsin }}(t)=I_{h 1} \sin (\omega t)+T \mathrm{HD}_{k} \cdot I_{h 1} \cdot \sin \left(k \omega t+\varphi_{k}\right)
$$

where $\omega=2 \pi f$ and $f=50 \mathrm{~Hz}$.

\section{HARMONIC AC LOSS IN HTS TAPE CARRYING CURRENT WITH LOW ORDER HARMONICS}

\section{A. Effect of harmonic phase angle on AC losses}

Figs. 1(a)-(c) report AC losses in HTS tape carrying nonsinusoidal current which is distorted by the $3^{\text {rd }}, 5^{\text {th }}$, and $7^{\text {th }}$ harmonics, respectively, illustrated against the phase angle $\varphi$ of current harmonic ranging from $0^{\circ}$ to $360^{\circ}$, at identical $T H D=$ 0.2 , with $i_{\mathrm{m}}$ varies from 0.2 to 0.5 . As it is shown in Figs. 1(a)(c), nonsinusoidal AC loss curves is symmetrical at $\varphi=180^{\circ}$, when the current is distorted by the $3^{\text {rd }}$, the $5^{\text {th }}$, and the $7^{\text {th }}$ harmonic orders with different phase angles.

At a given phase angle and harmonic order, nonsinusoidal AC loss increases with the increase of $i_{\mathrm{m}}$, when the current is distorted by the $3^{\text {rd }}$, the $5^{\text {th }}$ or the $7^{\text {th }}$ harmonic. This is due to stronger perpendicular magnetic field caused by higher $i_{\mathrm{m}}$, as explained in [16].

Nonsinusoidal AC loss reaches the minimum at $\varphi=0^{\circ}$ and the maximum at $\varphi=180^{\circ}$, in Fig. 1(a) and (c), when tape carries current with the $3^{\text {rd }}$ and the $7^{\text {th }}$ harmonic, at a given $i_{\mathrm{m}}$ and $T H D$ $=0.2$. When $0^{\circ}<\varphi<180^{\circ}, \mathrm{AC}$ loss increases with the increase of $\varphi$. The AC loss curve for the $5^{\text {th }}$ harmonic order in Fig. 1(b)
TABLE I

SPECIFICATIONS OF HTS TAPE

\begin{tabular}{ccc}
\hline \hline Parameter & Value & Unit \\
\hline Thickness of superconducting layer $\left(t_{\mathrm{sc}}\right)$ & 1 & $\mu \mathrm{m}$ \\
Width of tape $\left(w_{\text {tape }}\right)$ & 3 & $\mathrm{~mm}$ \\
Critical current density $\left(J_{\mathrm{c} 0}\right) @ 77 \mathrm{~K}$ & 1 & $\mathrm{MA} / \mathrm{cm}^{2}$ \\
$E-J$ power law factor $(n)$ & 25 & --- \\
\hline \hline
\end{tabular}

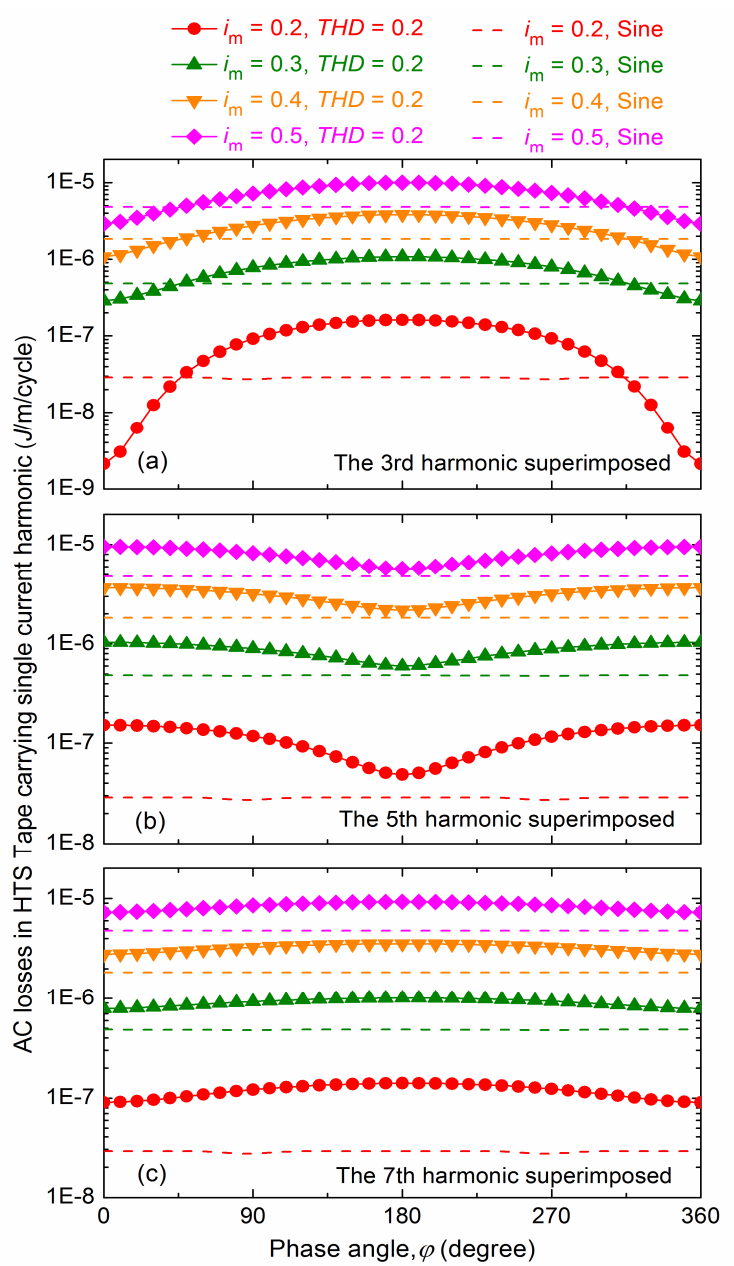

Fig. 1. AC losses in HTS tape carrying distorted current with different $i_{m}$, plotted against phase angle $\varphi$ of current harmonics: the $3^{\text {rd }}$, the $5^{\text {th }}$, and the $7^{\text {th }}$ harmonics at $T H D_{k}=0.2$.

is opposite to the trend for the $3^{\text {rd }}$, and $7^{\text {th }}$ orders, i.e. nonsinusoidal $\mathrm{AC}$ losses reach the minimum and maximum at $\varphi=180^{\circ}$ and $0^{\circ}$, respectively.

In Figs. 1(b) and (c), nonsinusoidal AC loss is always higher than sinusoidal AC loss, when current is distorted with the $5^{\text {th }}$ and the $7^{\text {th }}$ harmonic at any phase angle and at fixed $T H D=0.2$. Nonsinusoidal AC loss in Fig. 1(a), however, is higher than sinusoidal AC loss, when $50^{\circ}<\varphi_{3}<310^{\circ}$ while it is smaller than sinusoidal AC loss, when $\varphi_{3}<50^{\circ}$ or $\varphi_{3}>310^{\circ}$. This is due to the fact that superimposing a current harmonic to the sinusoidal current alters the current waveform, and thus, either strengthens or weakens the peak value of total resultant nonsinusoidal current.

Fig. 2 shows four nonsinusoidal current waveforms distorted by the $3^{\text {rd }}$ harmonic when $T H D_{3}=0.2$, at $\varphi_{3}=0^{\circ}, 30^{\circ}, 90^{\circ}$, and $180^{\circ}$. The sinusoidal current waveform was plotted as well in per-unit, as a guideline. The maximum peak of waveforms 


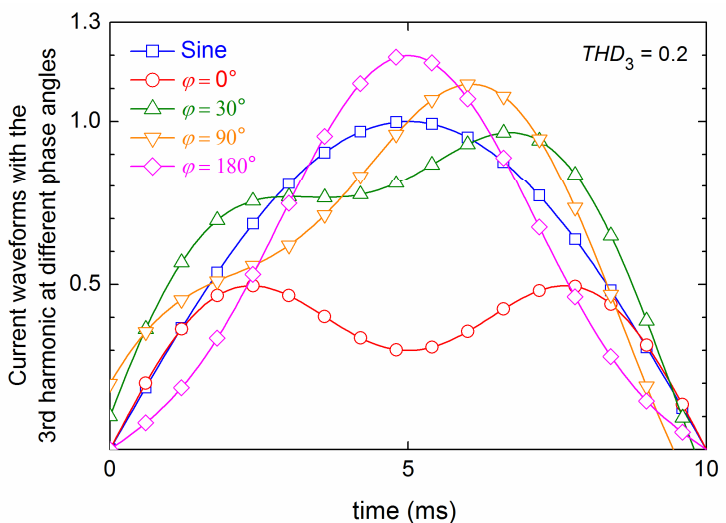

Fig. 2. Nonsinusoidal current waveforms with the $3^{\text {rd }}$ harmonic at $T H D_{3}=0.2$, and different phase angles, $\varphi=0^{\circ}, 30^{\circ}, 90^{\circ}$, and $180^{\circ}$, plotted together with sinusoidal current waveform in per-unit scale

follows a relation, $I_{\max , \varphi 3=0}<I_{\max , \varphi 3}=30<I_{\max , \text { sine }}<I_{\max , \varphi 3}=90$

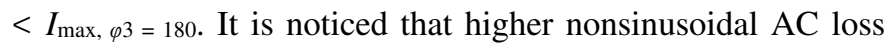
occurs at phase angle which leads to higher current peak.

\section{B. Effect of THD on harmonic AC losses}

Fig. 3(a)-(c) show the nonsinusoidal AC losses in HTS tape carrying distorted current with the $3^{\text {rd }}$, the $5^{\text {th }}$, and the $7^{\text {th }}$ harmonics, at $i_{\mathrm{m}}=0.3$ and $\varphi$ ranges from $0^{\circ}$ to $180^{\circ}$, plotted versus $T H D$ values. Here, only $0^{\circ}<\varphi<180^{\circ}$ was considered, due to the aforementioned symmetry in results.

An interesting phenomenon was observed in Fig. 3(a), that when $0^{\circ} \leq \varphi_{3} \leq 60^{\circ}$, there exists a minimum AC loss value at a specific $T H D$. This minimum occurs at $T H D_{3}=0.2$ for $\varphi_{3}=0^{\circ}$, which agrees with the finding in our previous paper [16]; at $\varphi_{3}$ $=30^{\circ}$, the minimum loss occurs at $T H D=0.1$. This shows that with the increase of $\varphi_{3}$, this minimum loss displaces towards lower THDs. Actually, it is inferred that when $\varphi_{3}$ is low, there will be always such a minimum AC loss value at a certain $T H D$ level. When $60^{\circ}<\varphi_{3}<300^{\circ}$, AC loss monotonically increases with the increase of $T H D$.

At $\varphi_{5}=180^{\circ}$ in Fig. 3(b), nonsinusoidal AC loss reduces first as $T H D_{5}$ increases from 0 ; then there appears a minimum $\mathrm{AC}$ loss when $T H D_{5}$ is around 0.1 ; after a certain $T H D_{5}, \mathrm{AC}$ loss monotonically increases with the increase of $T H D_{5}$. When $\varphi_{5}<$ $150^{\circ}$, AC loss monotonically increases versus $T H D$. In Fig. 3(c), however, nonsinusoidal AC loss value monotonically increases with increase of $T H D_{7}$, at any phase angle.

An overview of Figs. 3(a)-(c) was drawn that, the AC loss curves versus $T H D$ at different phase angles are much more scattered for the $3^{\text {rd }}$ harmonics while it is less scattered in case of the $7^{\text {th }}$ harmonics. This indicates at a given THD level, nonsinusoidal AC loss result in HTS tape get less affected by the phase angle of the $7^{\text {th }}$ harmonic order than the $3^{\text {rd }}$.

\section{HARMONIC AC LOSS IN HTS TAPE WITH CURRENT HARMONIC ORDER BELOW $20^{\mathrm{TH}}$}

In many large scale power applications, higher order harmonics appear in current waveform. For most of these cases, especially for grid connected devices, current harmonics below $1 \mathrm{kHz}$ (below the $20^{\text {th }}$ order), must be considered. It should be

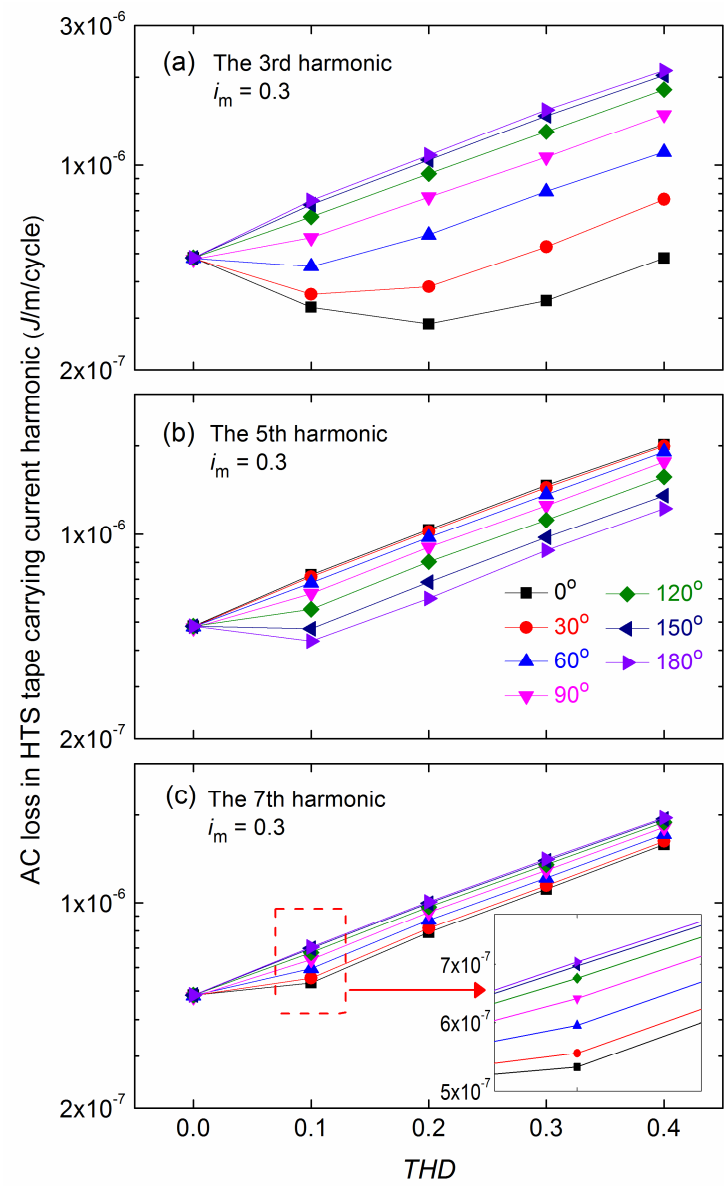

Fig. 3. AC losses in HTS tape carrying distorted current with the $3^{\text {rd }}$, the $5^{\text {th }}$, and the $7^{\text {th }}$ harmonics plotted against different $T H D$ s, at $i_{\mathrm{m}}=0.3$ and different $\varphi$

mentioned that the phase angle of the harmonics is practically found by applying Fourier Transform to the time domain current waveforms, and is not an independent parameter.

Fig. 4 shows the nonsinusoidal AC losses in HTS tape carrying current harmonics with orders below the $20^{\text {th }}$, plotted versus phase angle at $i_{\mathrm{m}}=0.5$ and $T H D_{i}=0.3$, and compared with sinusoidal AC loss results. It was observed that AC losses in tape carrying harmonic current with harmonic order of $\{5,7$, $9,11,13,15,17,19\}$, are much higher than the sinusoidal AC loss at any phase angle. However, AC loss in tape carrying the $3^{\text {rd }}$ harmonic is lower than sinusoidal AC loss, when $0^{\circ}<\varphi_{3}<$ $25^{\circ}$ and $335^{\circ}<\varphi_{3}<360^{\circ}$; whilst $\mathrm{AC}$ loss is higher than sinusoidal loss when $25^{\circ}<\varphi_{3}<335^{\circ}$.

It is found that the maximum nonsinusoidal AC loss in HTS tape appears at $\varphi=180^{\circ}$ for harmonic order $k=\{3,7,11,15$, $19\}$, and at $\varphi=0^{\circ}$ for harmonic order $k=\{5,9,13,17\}$. On the contrary, the minimum nonsinusoidal AC loss in HTS tape appears at $\varphi=0^{\circ}$ for $k=\{3,7,11,15,19\}$, and at $\varphi=180^{\circ}$ for harmonic order $k=\{5,9,13,17\}$. Assuming $k=(2 m-1)$, where $m=\{2,3,4, \ldots\}$, the maximum AC loss occurs at $\varphi=0^{\circ}$ if $m$ is an odd number, and at $\varphi=180^{\circ}$ if $m$ is an even number.

It was also observed in Fig. 4, as the harmonic order increases, nonsinusoidal AC losses in HTS tape converge towards a constant value, although the phase angle varies. It indicates AC loss gets insusceptible to phase angle at higher harmonic orders. This phenomenon is further illustrated in Fig. 5, which shows 


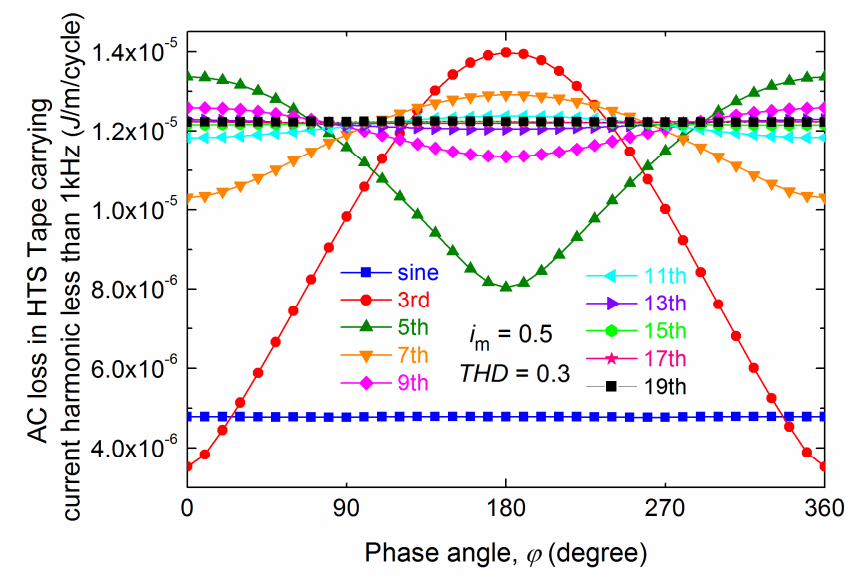

Fig. 4. Calculated harmonic AC losses in HTS tape carrying current harmonics below $20^{\text {th }}$, plotted against phase angle, at $i_{\mathrm{m}}=0.5$ and $T H D_{\mathrm{i}}=0.2$.

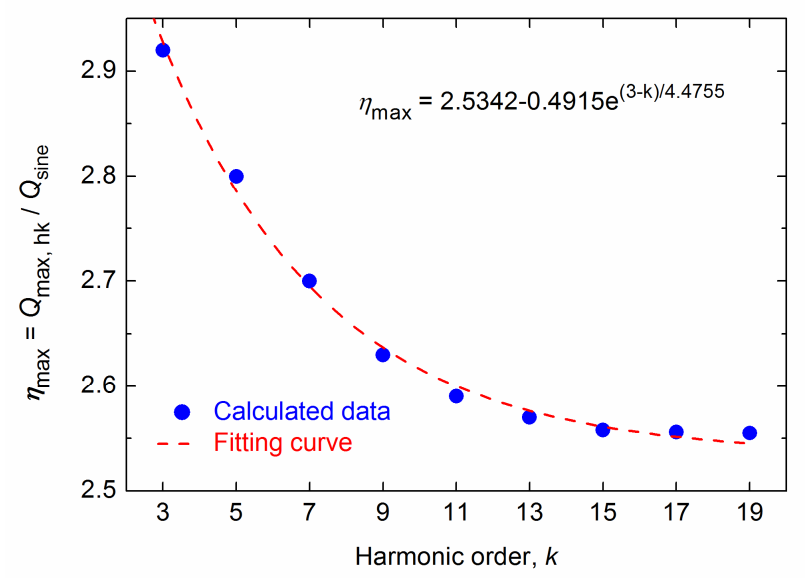

Fig. 5. The variation of ratio of nonsinusoidal AC loss in respect to sinusoidal one for harmonic orders below $1 \mathrm{kHz}$ at $i_{\mathrm{m}}=0.5$ and $T H D_{\mathrm{i}}=0.3$.

the $\eta_{\max }=Q_{\max , \mathrm{hk}} / Q_{\text {sine, }}$, ratio of the maximum nonsinusoidal AC loss under each harmonic order, $Q_{\mathrm{max}, \mathrm{hk}}$, to sinusoidal AC loss value, $Q_{\text {sine, at }} i_{\mathrm{m}}=0.5$ and $T H D=0.3$, where $k=\{3,5,7$, $9,11,13,15,17,19\}$. As shown in Fig. 5, $\eta_{\max }$ decreases from around 3 to 2.55, when harmonic order increases from 3 to 19 . Fig. 4 and the exponential fit in Fig. 5, clearly show that when the harmonic order exceeds $15, Q_{\max , \mathrm{hk}}$ and $\eta_{\max }$ tends to get constant. This is to say, at higher harmonic orders, the maximum nonsinusoidal AC loss is less dependent on harmonic order. The loss ratio for higher order harmonics tends to be about 2.537 based on curve fitting data in Fig. 5, indicating the dependency of AC loss of HTS tape to phase angle as well as the harmonic order itself reduces at higher order harmonics.

This is a very important finding for thermal load estimation in cooling system design (especially considering existing technology of cryocoolers with penalty factors of about 15 at $77 \mathrm{~K}$, and 100 at $20 \mathrm{~K}$ respectively) [5-6], since it reduces the design complexity when it comes to AC loss calculation for final heat load estimation. In addition, it simplifies the nonsinusoidal AC loss calculation task, when one considers a wide range of harmonics in superconducting windings. Nonsinusoidal AC loss could be predicted by a constant value for higher order harmonics above $1 \mathrm{kHz}$, even as a pessimistic design. The argument is at higher order harmonics, though the

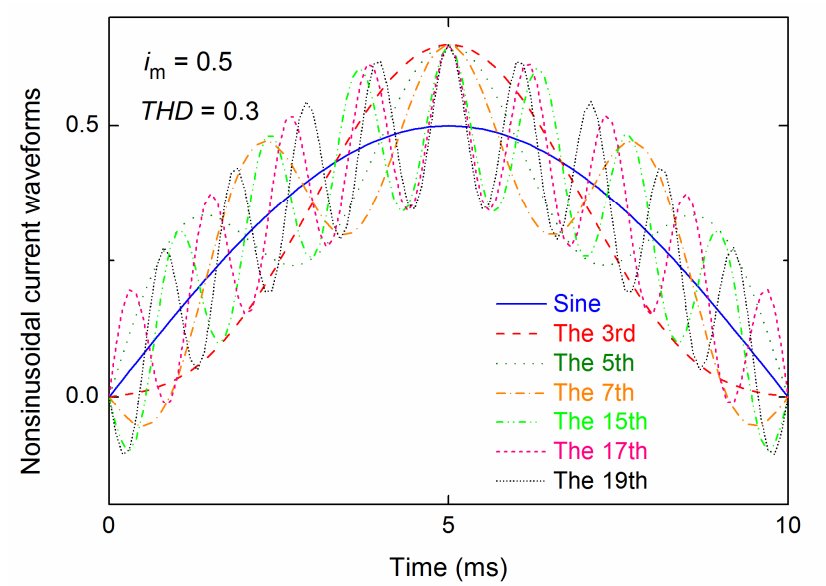

Fig. 6. Applied nonsinusoidal current containing harmonic orders below $20^{\text {th }}$ at $\mathrm{i}_{\mathrm{m}}=0.5, T H D_{\mathrm{i}}=0.3$, and phase angle correspondent to the maximum AC loss.

TABLE II

HARMONIC SPECTRUM OF DISTORTED CURRENT WAVEFORM

\begin{tabular}{ccc}
\hline \hline Harmonic order & Amplitude $(\%)$ & Phase angle $\left(^{\circ}\right)$ \\
\hline $1^{\text {st }}$ & 100 & -26 \\
$5^{\text {th }}$ & 25 & -94 \\
$7^{\text {th }}$ & 17 & -67 \\
$11^{\text {th }}$ & 9 & -67 \\
$13^{\text {th }}$ & 5 & 32 \\
$T H D \%$ & & -46 \\
\hline \hline
\end{tabular}

TABLE III

THE RESULTS OF AC LOSS CALCULATION FOR DISTORTED CURRENT SPECTRUM

\begin{tabular}{cc}
\hline \hline Scenario no. & AC loss $(\mathrm{J} / \mathrm{m} / \mathrm{cycle})$ \\
\hline \#1 Scenario & $4.78 \mathrm{E}-6$ \\
\#2 Scenario & $7.69 \mathrm{E}-6$ \\
\#3 Scenario & $1.55 \mathrm{E}-5$ \\
Loss ratio (\#2 to \#1 Scenario) & 1.61 \\
Loss ratio (\#3 to \#2 Scenario) & 2.02 \\
Loss ratio (\#3 to \#1 Scenario) & 3.24 \\
\hline \hline
\end{tabular}

number of peaks in each cycle of applied current increases, the waveform of currents gets more similarly distorted as depicted in Fig. 6. At higher order harmonics, the distorted waveforms are modulated very rapidly and converging towards same peak. In other words, the harmonic current becomes a ripple on the fundamental waveform rather than a huge change in waveform itself.

\section{EFFECT OF PHASE ANGLE OF HARMONICS IN A DISTORTED CURRENT SPECTRUM ON AC LOSS}

This section demonstrates how AC loss results could be affected, if the phase angle of current harmonics is not well considered. Three scenarios were chosen and compared to achieve this goal: \#1) pure sinusoidal current. \#2) a current harmonic spectrum, but all harmonic orders are in-phase with fundamental order. \#3) a current harmonic spectrum considering phase angle of each individual harmonic order.

For this purpose, a real current spectrum was considered. Table II shows the harmonic spectrum of a transformer current waveform which supplies plug-in electric vehicle chargers [23]. This current harmonic spectrum was applied to the HTS tape under the three aforementioned scenarios, and the sinusoidal and nonsinusoidal AC losses were calculated and reported in 
Table III. As it can be seen from Table III, nonsinusoidal AC loss of both \#2 and \#3 scenarios when current is distorted is much higher than sinusoidal AC loss. In addition, with/without considering the harmonic phase angle, AC loss increases by 3.24 and 2.02 times of that of sinusoidal value. It is found that conventional harmonic analysis modeling approach without considering phase angle of each harmonic order leads to a tremendous underestimation of $\mathrm{AC}$ loss value, and as a consequence, underestimation of heat load for cooling system.

\section{CONCLUSION}

In this work, we numerically investigated the AC loss characterization in HTS tape carrying harmonic currents with orders below $20^{\text {th }}$, considering the phase angles $\varphi$ varies from 0 to $2 \pi$, based on $H$-formulation in COMSOL Multiphysics. Effect of harmonic amplitude and THD on AC loss behavior were also considered. The conclusions are summarized as follows:

1) Different phase angles change the maximum amplitude of the resultant nonsinusoidal current. When the maximum amplitude of the resultant current is bigger, nonsinusoidal AC loss in HTS tape is higher in that corresponding phase angle.

2) The nonsinusoidal AC loss might be lower than that of sinusoidal, when the phase angle falls in a certain range.

3 ) In case of nonsinusoidal current with $3^{\text {rd }}$ harmonics, with the increase of $\varphi_{3}$, the minimum loss displaces towards lower THDs. But meeting this minimum is also phase angle dependent.

4) Assuming the harmonic order $k=(2 m-1)$, where $k<20$ and $m=\{2,3,4,5,6,7,8,9,10\}$, at a given harmonic amplitude and $i_{\mathrm{m}}$, the maximum nonsinusoidal AC loss appears at $\varphi=180^{\circ}$ if $m$ is an even number, and at $\varphi=0^{\circ}$ if $m$ is an odd number, when HTS tape carrying a single current harmonic component.

5) At higher current harmonic orders, nonsinusoidal AC loss is less phase angle dependent, compared to lower orders. It indicates $\mathrm{AC}$ loss gets unsusceptible to phase angle at higher harmonic order.

This study investigates the effect of harmonic phase angle, harmonic orders up to the $19^{\text {th }}$, and harmonic amplitude on AC losses of HTS coated conductor, when the current contains one harmonic component, and also builds a fundamental understanding on the influencing aspects of nonsinusoidal AC loss. In the future, we will carry out the AC loss investigation of current spectrum which consists of multiple harmonic components.

\section{REFERENCES}

[1] K. S. Haran, S. Kalsi, T. Arndt, H. Karmaker, R. Badcock, B. Buckley, T. Haugan, M. Izumi, D. Loder, J. W. Bray, P. Masson, and E. W. Stautner, "High power density superconducting machines - development status and technology roadmap," Supercond. Sci. Technol., vol. 30, no. 12, 2017, pp. 1-30.

[2] M. Yazdani-Asrami, M. Staines, G. Sidorov, M. Davies, J. Bailey, N. Allpress, N. Glasson, and S. Asghar Gholamian, "Fault current limiting HTS transformer with extended fault withstand time," Supercond. Sci. Technol., vol. 32, no. 3, pp. 1-13, 2019.

[3] W. Song, X. Pei, J. Xi, and X. Zeng, "A Novel Helical Superconducting Fault Current Limiter for Electric Propulsion Aircraft," IEEE Trans. Transp. Electrification, Early Access, pp. 1-11, 2020.
[4] F. Gomory, and J. Sheng, "Two methods of AC loss calculation in numerical modelling of superconducting coils," Supercond. Sci. Technol., vol. 30, no. 6, pp. 1-12, 2017.

[5] J. Palmer, and E. Shehab, "Modelling of cryogenic cooling system design concepts for superconducting aircraft propulsion", IET Electrical Systems in Transportation, vol. 6, no. 3, 2016, pp. 170-1788.

[6] R. Radebaugh, "Refrigeration for Superconductors", IEEE Trans. Appl. Supercond., vol. 21, no. 3, 2011, pp. 3273-3276.

[7] M. Yazdani-Asrami, M. Mirzaie, and A. Akmal, "No-load loss calculation of distribution transformers supplied by nonsinusoidal voltage using three dimensional finite element analysis," Energy, vol. 50, pp. 205-219, 2013.

[8] M. Yazdani-Asrami, M. Mirzaie, and A. Akmal, "Investigation on impact of current harmonic contents on the distribution transformer losses and remaining life," in Proc. IEEE Int. Conf. Power Energy, 2010, pp. 689-694.

[9] S. Venuturumilli, F. Berg, L. Prisse, M. Zhang, and W. Yuan, "DC Line to Line Short-Circuit Fault Management in a Turbo-Electric Aircraft Propulsion System Using Superconducting Devices", IEEE Trans. Appl. Supercond., vol. 29, no. 5, 2019, pp. 1-6.

[10] H. Alafnan, M. Elshiekh, X. Pei, S. Altouq, S. M. Fazeli, Q. Sun, M. Zhang, and W. Yuan, "Application of SMES-FCL in Electric Aircraft for Stability Improvement", IEEE Trans. Appl. Supercond., vol. 29, no. 5, 2019, pp. 1-6.

[11] G. Furman, M. Spektor, V. Meerovich, and V. Sokolovsky, "Losses in coated conductors under non-sinusoidal currents and magnetic fields", $J$. Supercond. Nov. Magn., vol. 24, no.1-2, 2011, pp. 1045-1051.

[12] Z. Zhu, Y. Wang, S. Venuturumilli, J. Sheng, M. Zhang, and W. Yuan, "Influence of Harmonic Current on Magnetization Loss of a Triaxial CORC REBCO Cable for Hybrid Electric Aircraft," IEEE Trans. Appl. Supercond., vol. 28 , no. 4 , pp. 1-5, 2018.

[13] B. Shen, C. Li, J. Geng, X. Zhang, J. Gawith, J. Ma, Y. Liu, F. Grilli, and T. A. Coombs, "Power dissipation in HTS coated conductor coils under the simultaneous action of AC and DC currents and fields," Supercond. Sci. Technol., vol. 31, no. 7, pp. 1-12, 2018.

[14] B. Shen, C. Li, J. Geng, Q. Dong, J. Ma, J. Gawith, K. Zhang, Z. Li, J. Chen, W. Zhou, X. Li, J. Sheng, Z. Li, Z. Huang, J. Yang, and T. A. Coombs, "Power Dissipation in the HTS Coated Conductor Tapes and Coils Under the Action of Different Oscillating Currents and Fields," IEEE Trans. Appl. Supercond., vol. 29, no. 5, pp. 1-5, 2019.

[15] W. Song, J. Fang, and Z. Jiang, "Numerical AC Loss Analysis in HTS Stack Carrying Non-sinusoidal Transport Current," IEEE Trans. Appl. Supercond., vol. 29, no. 2, pp. 1-5, 2019.

[16] M. Yazdani-Asrami, W. Song, X. Pei, M. Zhang, and W. Yuan, "AC Loss Characterization of HTS Pancake and Solenoid Coils Carrying Nonsinusoidal Currents," IEEE Trans. Appl. Supercond., vol. 30, no. 5, pp. 1-9, 2020.

[17] M. Yazdani-Asrami, S. A. Gholamian, S. M. Mirimani, and J. Adabi, "Calculation of AC Magnetizing Loss of ReBCO Superconducting Tapes Subjected to Applied Distorted Magnetic Fields," J. Supercond. Nov. Magn., vol. 31, no.12, 2018, pp. 3875-3888.

[18] Z. Hong, and T. A. Coombs, "Numerical Modelling of AC Loss in Coated Conductors by Finite Element Software Using H Formulation," J. Supercond. Nov. Magn., vol. 23, 2010, pp. 1551-1562.

[19] R. Brambilla, F. Grilli, and L. Martini, "Development of an edge-element model for AC loss computation of high-temperature superconductors," Supercond. Sci. Technol., vol. 20, no. 1, pp. 16-24, 2007.

[20] F. Grilli, "Numerical Modeling of HTS Applications", IEEE Trans. Appl. Supercond., vol. 26, no. 3, 2016, pp. 1-8.

[21] V. M. Rodriguez-Zermeno, N. Mijatovic, C. Traholt, T. Zirngibl, E. Seiler, A. B. Abrahamsen, N. F. Pedersen, and M. P. Sorensen, "Towards Faster FEM Simulation of Thin Film Superconductors: A Multiscale Approach", IEEE Trans. Appl. Supercond., vol. 21, no. 3, 2011, pp. 3273-3276.

[22] R. Brambilla, F. Grilli, L. Martini, and F. Sirois, "Integral equations for the current density in thin conductors and their solution by the finite element method," Supercond. Sci. Technol., vol. 21, no. 10, pp. 1-8, 2008.

[23] M. A. S. Masoum, P. S. Moses, and S. Deilami, "Load Management in Smart Grids Considering Harmonic Distortion and Transformer Derating", IEEE 2010 Innovative Smart Grid Technologies (ISGT2010), 2010, pp. 1-7. 